Journal of Adhesion Science and Technology, 2012, vol. 26(1-3) p. 189-205

\title{
Insights into Surface Treatment Methods of Titanium Dental Implants
}

\author{
Cecilia Yan Guo, Alexander Tin Hong Tang and Jukka Pekka Matinlinna* \\ Faculty of Dentistry, University of Hong Kong \\ 34 Hospital Road, Sai Ying Pun, Hong Kong
}

\begin{abstract}
Titanium is the most widely used material for dental implants due to its desirable properties, e.g. high biocompatibility, low density, high stiffness and strength, etc. More importantly, titanium implants may osseointegrate with living bone, meaning that new bone grows directly onto the surface of the implant, without any intermediate soft tissue layer. A successfully osseointegrated implant generally has a strong bonding to the adjacent bone; consequently, it usually functions well and remains stable for long service period. It also has been clinically proven that surface treatment methods can improve the rate and quality of titanium implants' osseointegration. This article focuses on two such

\footnotetext{
* To whom correspondence should be addressed. Department of Dental Materials Science, Faculty of Dentistry, University of Hong Kong, 34 Hospital Road, Sai Ying Pun, Hong Kong. Tel: +852-28590380; Fax: +852-25489464; email: jpmat@hku.hk.
} 
methods, i.e. surface roughening and hydroxyapatite (HA) coating. In addition, we discuss a promising new methodology, which attempts to modify the surface charge of titanium materials. This paper focuses on the current best surface treatment methods for titanium dental implants developed and improved in the past two decades, i.e., 1990-2011.

Key words: titanium, dental implant, surface treatment, osseointegration

\section{Introduction}

Human teeth may fail irreversibly. When this happens, it is possible to restore most of the functions of the failed teeth with dental implants. A dental implant is an artificial tooth root that supports restorations resembling a tooth or a group of teeth (i.e., a denture). Nowadays, titanium is the most widely used material for the manufacturing of dental implants, due to the following reasons. First, titanium has several desirable mechanical properties. For instance, titanium is biomechanically very strong and has high strengthto-weight ratio as well as corrosion resistance. In particular, the tensile strength of titanium alloys is comparable to many materials, including iron-based super-alloys, and at the same time, titanium alloys are much lighter than these materials [1, 2]. Therefore, compared to dental implants built with other materials, titanium dental implants are significantly lighter, more durable, and capable of withstanding higher pressure without 
harmful deformation, e.g., cracking. Second, titanium is highly biocompatible, as a result of low-toxicity and a low rate of ion-release from its surface $[3,4]$. Such properties are widely understood to be the consequence of an inert surface oxide film. When pure titanium or its alloys are exposed to air, a layer of titanium dioxide with a thickness of approximately 2-5 nm can often be formed in a few seconds. This thin film also protects the titanium materials, making the latter highly resistant to corrosion.

Third and most importantly, titanium may osseointegrate [5] with living bone. Specifically, an implant usually takes the form of a screw, which naturally resists pull-out forces well. However, screws tend to loosen in the presence of torsion (i.e. "unscrewing" forces), and the only way to secure them in place is to ensure a strong bonding between the implant and the adjacent bones [6]. Osseointegration is one such strong binding, in which new bone forms directly onto the surface of the implant, without any intermediate layers of scar tissues, cartilage, or fibers. After osseointegration has completed, the implant is not only accepted by the host bone, but the two also form direct structural and functional connections [7]. Consequently, stress acting on the implant can be transferred efficiently to the host bone, without any relative motion occurring at the interface. The resulting displacement between the dental implant and the host bone is restricted to atomic distances [6]. Hence, a well osseointegrated titanium dental implant is highly 
stable, and can remain in place for long periods, even lifetime.

However, not all titanium dental implants osseointegrate well in vivo. The boneimplant bonding can be too weak to hold the implant in place. The implant may fail to osseointegrate and loosen soon after surgical insertion. Hence, strong and rapid ossointegration is pivotal to the success of titanium dental implantation. To achieve this, it is important that (i) a large amount of bone-forming cells (osteoblasts) adhere to the dental implant surface. These cells interact with the implant surface to develop a socalled "bone-specific extracellular matrix", which later mineralizes to form an integrated bone / implant interface; (ii) relatively few fiber-forming cells (fibroblasts) should attach to the surface of the implant, since they form soft tissues, which negatively affects the bone-implant bonding. The growth of both osteoblasts and fibroblasts seems to depend on surface condition of the implant [8]. Although the precise role of surface properties on osseointegration remains poorly understood, previous research has found that several properties, e.g. surface topography, chemical composition, cleanliness and electrical charges, etc., have significant impacts on the quality and rate of the implant's osseointegration [1].

Recently, research on surface treatment methods for titanium dental implants has achieved considerable success in improving and accelerating their osseointegration. This 
work aims at summing up the state of the art techniques for this purpose. In the following, Section 1.1 describes the methodology for this review. Sections 2 and 3 of this paper summerises two common approaches, i. e. surface roughening and HA coating. Section 4 discusses an emerging methodology that modifies the surface charge of the implant. Finally, Section 5 summarizes the titanium surface treatment methods reviewed in this paper, and discusses promising directions for future investigations.

\subsection{Methodology of the Review}

Our search strategy gives priority to methods with clinically proven effectiveness, especially those with good results on Asian patients. In the meantime, we also search for promising new approaches that have drawn significant research attention, which may be applied clinically in the near future, or integrated into existing solutions.

We classify the surface treatment methods found in the literature into three categories: surface roughening, surface coating, and emerging techniques. Among the various emerging techniques, we include the one (i.e., surface charge modification) that has attracted most attention among dental materials researchers. For each category, we present first its theoretical foundation, followed by descriptions of the most successful methods in this category. The strengths and limitations of each method are also discussed, 
and relative merits and drawbacks of different methods are analyzed, whenever possible.

\section{Surface Roughening}

A machined titanium dental implant without any surface treatment usually has a relatively smooth surface. On the other hand, studies have shown that titanium materials with rough surfaces osseointegrate better $[9,10]$, in terms of both quality and rate, than the ones with smooth surfaces. The effects of surface roughness on osseointegration from a theoretical point of view will be explained. Four popular titanium surface roughening methods, namely titanium plasma spray (abbreviated as TPS), sandblasting, acid-etching, and sandblasting by large grits followed by acid-etching (abbreviated as SLA) will be detailed in section 2.2 .

\subsection{Impact of Surface Roughness}

Roughness is a type of topographical property of a given surface. Depending on the area of the given surface, we may classify three levels of roughness; macrotopographic, microtopographic, and nano-level of roughness. The first kind of roughness concerns relatively a large surface area; in particular, a macrotopographically rough surface contains spikes whose statistical average height (Sa value) is more than $10 \mu \mathrm{m}$ [1]. In contrast, microtopographical roughness deals with smaller surface areas. The surface has 
spikes of height $1 \mu \mathrm{m} \leq \mathrm{Sa}<10 \mu \mathrm{m}$. Finally, the nano-level roughness concerns even finer surface areas, with spike height less than $1 \mu \mathrm{m}$, usually in the nanometer range. Different levels of roughness are independent of each other. A surface can be simultaneously smooth on the macrotopographic level and rough on the microtopographic level. Fig. 1 illustrates four different levels of surface roughness.

Since a titanium dental implant usually takes the form of a screw, it already has a certain degree of macrotopographical roughness due to peaks and valleys of the screw thread. Meanwhile, an unmodified titanium dental implant is considered to have a smooth surface on the microtopographical as well as nano-sized level [11]. Increasing roughness of the implant on any of the three levels helps its osseointegration [1]. The precise reason for this phenomenon is only poorly understood. A number of factors may play significant roles. First, a rough surface has larger surface area than a smooth one; consequently, with a rough surface, a higher number of host cells, including both osteoblasts and fibroblasts, get into contact with the implant surface [12]. Second, for unknown reasons, surface roughness helps osteoblasts more than fibroblasts to attach to and live on the implant surface [13], leading to more bone and less fiber formed between the host bone and the rough implant surface. Third, from a mechanical point of view, a rough surface provides stronger interlocking between the implant and the newly formed bone, improving the 
long-term stability of the implant [12].

A macro-porosity with pore size of $150 \mu \mathrm{m}$ in diameter is similar to the dimensions of the lacunae of human spongy bone, and, therefore, is considered ideal for all kinds of tissue ingrowth [14]. Microtopographical and nano-size roughnesses, on the other hand, are more important for increasing the number of osteoblasts attachment to the surface [15, 16], since the osteoblasts can better orient themselves into the smaller grooves [17]. In addition, microtopographical and nano-sized roughnesses are beneficial to the interaction between osteoblasts and the implant in many aspects, including their proliferation, differentiation and bone production [18]. Previous works draw the conclusion that microtopographical roughness leads to the highest bone-implant contact and torsion resistance among all three levels of surface roughness [19, 20]. Wennerberg et al. reported that the optimal surface roughness for implants is around Sa $=1.5 \mu \mathrm{m}$; higher or lower values for Sa were found to cause a weaker bone response [20].

However, a high macrotopographical roughness of the implant surface has two notable adverse effects: bacterial infection around the implant, i.e., peri-implantitis, and ionic leakage from the implant [21]. In addition, nano-sized roughness, though important for osseointegration, is difficult to obtain using current methods [16]. Overall, increasing microtopographical roughness is currently the most important goal in surface roughness 
modification, which may be achieved by effective methods as follows.

\subsection{Titanium Plasma Spray (TPS)}

Plasma spraying is considered to be the most popular coating technique. In this process, a gas plasma stream is first created by having an electrical arc between a finger-type tungsten cathode and a nozzle-type copper anode inside the plasma torch. Then, the process feeds the desired coating material power into this gas plasma stream, in order to melt and accelerate the power, and attach it to the substrate. This forms the desired lamellar structured coating, with a certain level of roughness [22].

Titanium powder can be plasma sprayed onto the surface of the dental implant to increase the latter's surface roughness, which is a process commonly referred to as TPS.

High-quality titanium coating requires high working temperature of the spray gases, which is obtained by the plasma process and the automation of plasma-spray devices [22]. TPS creates a macrotopography on titanium implant surface. Figure 2 shows the visual characterization of the surface geometry of titanium plasma-sprayed implant.

TPS implant shows higher roughness than sandblasted (Section 2.3), acid-etched (Section 2.4) or HA-coated (Section 3) titanium implants, which is believed to be beneficial to bone ingrowth and osseointegration [11]. Klokkevold et al. reported that 
TPS dental implant shows a complex surface with particulate sizes in the range 1-25 $\mu \mathrm{m}$, and the particle density in the valleys normally appears higher than those on the thread peaks [11]. On the microtopographical level, however, the TPS implant surface is almost smooth, without much texture. They also compared TPS titanium implants with unmodified and dual acid-etched titanium implants (Section 2.4), and found that TPS implants exhibit the highest mean value of reverse torsion throughout the first three months after implantation [11].

However, the bonding between the coating and the titanium substrate is considered to be weak; as a result, its pull strength is similar to the HA-coated implants [23], which is not as strong as we describe in Section 3. In addition, as discussed in Section 2.1, the macrotopographical textures may lead to ion leakage from the implant. As a result, granules of titanium have been found to leak into the bone pocket adjacent to the TPS implants [24].

\subsection{Sandblasting}

The second surface treatment method, sandblasting, forces small grits in chosen shape and size across implant surfaces, usually by compressed air [25]. Sandblasted titanium surface has a rough and irregular microtopography, while retaining the 
macrotopographical and nano-level topographical properties of the original surface [26]. The specific degree of roughness of the sandblasted titanium surface depends on several controllable parameters [27]. These are the type and size of the grits, duration of blasting, air pressure, and distance between the source of the particles and the implant surface [27]. Apart from generating a macrotopographic roughness, sandblasting has the benefit of cleaning the implant surface and increasing its bioactivity [28]. Studies have confirmed that sandblasting a titanium implant surface significantly accelerates osteoblasts adhesion and proliferation [29], while at the same time compromises fibroblasts adhesion and proliferation [15]. Both facts promote implant’s osseointegration.

Two common choices of the sandblasting grits are aluminum trioxide (mostly $\mathrm{Al}_{2} \mathrm{O}_{3}$ ) and titanium dioxide $\left(\mathrm{TiO}_{2}\right)$. The former is more effective in terms of increasing surface roughness, since it is a harder material than $\mathrm{TiO}_{2} \cdot \mathrm{Al}_{2} \mathrm{O}_{3}$ is also inexpensive and widely available. However, $\mathrm{Al}_{2} \mathrm{O}_{3}$ grits often adhere to the implant surface after sandblasting, which are difficult to remove by subsequent cleaning steps, including ultrasonic cleaning [30], acid-etching [31] and sterilization [32]. This in effect decreases the purity of the titanium material [33], which leads to several undesirable drawbacks. First, $\mathrm{Al}_{2} \mathrm{O}_{3}$ has poor biocompatibility and bioactivity. Its presence adversely affects the implant's osseointegration [34]. Second, the decrease in titanium purity compromises its corrosion 
resistance and other mechanical merits [33]. Fortunately, the same study concluded that $\mathrm{Al}_{2} \mathrm{O}_{3}$ blasted titanium is still suitable as a dental implant material, in terms of chemical and mechanical properties [33].

$\mathrm{TiO}_{2}$ grits avoid the above drawbacks of $\mathrm{Al}_{2} \mathrm{O}_{3}$, since $\mathrm{TiO}_{2}$ is already present on the original titanium implant surface. However, it is also observed that $\mathrm{TiO}_{2}$ grits are as hard as the titanium surface; hence, $\mathrm{TiO}_{2}$ blasting is less effective than $\mathrm{Al}_{2} \mathrm{O}_{3}$ in increasing titanium surface roughness. $\mathrm{TiO}_{2}$ blasted implant surface only has a Sa value between 1$2 \mu \mathrm{m}$ [1]. Nevertheless, $\mathrm{TiO}_{2}$-blasted implants have been shown to effectively increase the durability and stability of titanium dental implant [35].

Besides $\mathrm{Al}_{2} \mathrm{O}_{3}$ and $\mathrm{TiO}_{2}$, bio-ceramics, such as $\mathrm{HA}$, have also been tested as sandblasting grits [36]. As explained in more detail in Section 3, HA is more biocompatible, and renders the titanium implant to osseointegrate better than $\mathrm{Al}_{2} \mathrm{O}_{3}$ and $\mathrm{TiO}_{2}$ roughened titanium implant body [37]. Therefore, theoretically, by leaving HA particles on the implant surface, HA-blasting is expected to improve the implant's osseointegration. Unfortunately, this technique is still in an experimental stage, and there are a number of technical difficulties in HA-blasting. For instance, HA particles have a weak bonding with the titanium substrate [36] and, thus, may be loosened from the implant surface over time. 


\subsection{Acid-Etching}

Acid-etching of titanium dental implant, by immersing it in strong acids (e.g., nitric acid, hydrochloric acid, hydrofluoric acid, sulfuric acid, and their blends) for a given period of time, creates a micro-roughness of $0.5-3 \mu \mathrm{m}$ with irregular pits of varying depth on titanium implant surface. At the same time, it cleans the implant surface, e.g., removes deposits, as a result of sandblasting. Several parameters affect the topography of etched implants, including the surface roughness or roughening procedure prior to etching, acid mixture, acid bath temperature, and etching time [38]. In practice, dual acid-etching is almost always used in which the titanium implant is immersed in a mixture of two strong acids (e.g., $\mathrm{HCl}$ and $\mathrm{H}_{2} \mathrm{SO}_{4}$ ). Fig. 3 shows the SEM images of unmodified, dual acidetched, and TPS treated titanium dental implant surfaces with magnifications of $\times 40$, $\times 100$ and $\times 1000$.

Acid-etching is often used in combination with sandblasting, as we shall describe in detail in Section 2.5. When used alone, it is known to accelerate osseointegration and improve the stability of the titanium dental implant at an early stage. It is found that in the first three months, the effectiveness of dual acid-etching, in terms of implant stability, is comparable to that of TPS [11]. On the other hand, the effects of acid-etching on the long-term stability of the titanium dental implant are rather limited [11]. 
A major drawback with acid-etching is that it causes hydrogen embrittlement, which leads to microcracks on the surface of the titanium dental implant [39]. Such cracks compromise the good mechanical properties, especially fatigue resistance of the titanium implant [39].

\subsection{SLA}

SLA method combines sandblasting and acid-etching. In SLA protocol, the titanium dental implant surface is first sandblasted with large grits, making the surface grossly rough. Then, the implant is acid-etched to form micro-pits on its surface. The resulting implant surface is both macrotopographically wavy and rough at the micro-level, which is believed to be beneficial for the implant osseointegration [40]. SLA modifies both the macrotopography and microtopography of the implant's surface. Within the microtopographical level, SLA leads to a rich, multi-tier texture. Fig. 4 shows the SLA implant surface. Observe that SLA introduces both fine pits (Fig. 4b) and higher-level roughened textures on top of these pits (Fig. 4a).

As a simple and effective technique, SLA has achieved considerable success. Titanium dental implants with and without SLA treatment have been compared experimentally [13]. It is found that significantly more osteoblasts (about 1.5 times), and 
fewer fibroblasts attach to the SLA implant compared with the one without SLA treatment. By comparing implants with SLA and simple acid-etching, it is reported that the former are over 30\% stronger than the latter in terms of torsion resistance, and $5 \%$ stronger in terms of pressure resistance [41]. In vivo study suggests that titanium implants with SLA surfaces allow a further reduction of healing time compared with titanium implants with TPS surfaces [24]. Fig. 5 shows the large amounts of bones attached to the SLA implant surface. This confirmed that after implantation, SLA titanium dental implant integrated well with the host bones. The arrows show the fractured bone trabecules and the bone ingrowth into the pits in (a) and (b) respectively.

Finally, studies have found that given a short healing period (3-6 weeks), SLAtreated titanium implants demonstrates even better osseointegration than ones coated with HA, which is far more complex and expensive process of implant coating [9, 42].

\section{Hydroxyapatite (HA) Coating}

\subsection{Hydroxyapatite}

HA $\left(\mathrm{Ca}_{10}\left(\mathrm{PO}_{4}\right)_{6}(\mathrm{OH})_{2}\right)$ is an inorganic compound found in the teeth and bones of the human body. In practice, artificially synthesized HA is commonly used as a biomaterial [14]. Compared with titanium, HA compounds are preferred in terms of biocompatibility. 
HA also osseointegrates faster and stronger than untreated titanium [43]. However, bulk

HA is relatively weak and brittle, and lacks the mechanical merits of titanium. Hence, HA is seldom used as the main material for dental implants. Instead, it is often coated on the surface of titanium implants. The resulting composite material combines the mechanical advantages of titanium and superior bioactivity and biocompatibility of HA, as explained in Section 3.2. Fig. 6 shows the SEM micrographs of a HA coated titanium surface.

Unlike titanium, HA has a porous structure. Therefore, HA naturally forms a rough surface, which is ideal for osseointegration as discussed in Section 2. This also means that no roughening treatment, such as sandblasting, is necessary for HA. Depending on the method used for synthesizing HA, the resulting material can be either micro-porous or macro-porous. Although both types of HA are capable of osseointegration, previous work has concluded that micro-porous HA is more preferable as the material for dental implants [14]. The main reasons are micro-porous HA has better mechanical properties, a lower rate of ion release, and host bones tend to heal faster with micro-porous HA [14].

\subsection{Hydroxyapatite-Coated Titanium Dental Implant}

HA is usually coated onto the surface of a titanium dental implant through plasma 
spraying, which is a similar process to TPS described in Section 2.2 [43]. Several alternatives, such as ion beam assisted deposition (IBAD), are also commonly used in practice. All these techniques are effective in producing high-quality coating [44, 45]. However, they require sophisticated and expensive equipment. Moreover, they all involve the use of high temperatures, making it difficult to control the coating composition and crystal structure, which are important for the overall quality of the dental implant as we shall explain soon [46, 47]. To overcome these problems, novel techniques have been proposed based on biomimetic processes, which produce HA coating in aqueous solutions at physiological temperatures [48].

Several studies found that HA-coated titanium dental implants osseointegrate faster and stronger than ones made of uncoated titanium [14, 49, 50]. They indicate that HA coating has two major advantages. First, faster osseointegration of HA coated on titanium dental implant leads to earlier stabilization of the implant in surrounding bone. Thus, healing time is reduced, and the final crown or bridge can be placed earlier on the implant. Second, stronger bonding between implant and bone extends the functional life of the implant-supported dental prosthesis. These benefits can greatly improve the success rate of dental implantation, especially in patients with poor bone qualities [43].

Numerous animal tests have been done to predict the human response to HA coated 
titanium dental implants [50-52]. In these tests, uncoated titanium materials, usually either untreated or sandblasted, were often used as control group to compare with HAcoated implants. The results indicate that HA-coated dental implants present notably better mechanical properties than uncoated ones in the early stage of healing after implantation. For instance, Cook et al. report that HA-coated implant improves the mean interface strength by 5-8 times compared to the uncoated, sandblasted titanium implant [50]. In the long-term, however, the advantages of coated titanium implants are less pronounced [43].

The quality of the HA coating depends on several key factors. The most important one is the thickness of the HA layer. Since titanium has much better mechanical properties than HA, the thinner the HA layer, the better the implant mechanically. Furthermore, a thin HA layer can preserve the porous structure of the HA surface more effectively [52]. Therefore, the HA layer should have the minimum thickness such that the coating does not dissolve [52]. Besides thickness, the choice of the HA material is also of significant importance. The HA coating layer slowly releases ions to surrounding tissues, which not only decreases the quality of implant / bone integration, but may also be harmful to the host tissues. The rate of ion release is negatively correlated with both the density and crystallinity of the HA material. Thus, the HA used for coating dental 
implants must reach a certain level of density and crystallinity [43].

Another important factor is the adhesion strength between HA coating and titanium implant. The properties of HA-coated titanium implants with weak adhesion strength can be worse than uncoated ones. The weakly bonded coating can be separated from the implant, which may lead to the disruption of the $\mathrm{TiO}_{2}$ layer on titanium surface, as well as momentary increase in titanium ion release [43]. Thus, the coating process should ensure that the HA layer is strongly bonded to the titanium substrate to maintain implant integrity as well to facilitate proper transmission of load from the implant to the surrounding bone. It has been shown that chemical composition and $\mathrm{Ca} / \mathrm{P}$ ratio of HA also seem to affect the behavior of HA coating [52].

Currently, HA-coated titanium material still faces several challenges. First, HA and titanium have very different physical properties. For example, there is a large gap between the thermal expansion coefficients of these two materials, meaning that increased temperature may lead to deformation of the implant surface. Variation in temperature during air transportation and autoclave sterilization may impart such stress to the implant coating. Second, there is evidence that after implantation, some HA coating on the implant could be resorbed [53]. This resorption has been observed to occur as early as 16 weeks after implantation [54]. Third, the bonding between HA coating and the 
metallic substrates is considered to be rather weak. Consequently, the coating may crack or separate from the substrates under heavy load, which further weakens the HA-bone bonding $[55,56]$. Eventually, the HA-bone may become too weak to support the titanium base implant, leading to the loss of the entire implant [57]. Fourth, the high biocompatibility of HA leads to a critical drawback, i.e., it provides a convenient

environment for bacteria to adhere to it. As a result, after implant placement, bacterial infection becomes a significant complication. Finally, study shows that HA fails to reach the expectation that new bones grow into the holes of its porous surface; instead, the authors find through autopsy that over $90 \%$ of those holes are filled by fibers [58].

\section{Surface Charge Modification}

Surface charge refers to the electric charge presents on a surface. The surface charge of a dental implant is caused by interactions between the aqueous environment in the mouth and the implant itself [8]. The surface charge of the implant has been shown to affect bone formation by inducing the differentiation of osteoblastic cells which form bone [59]. Compared to a neutral surface, a negatively-charged one promotes osteoblast adhesion by up to $60 \%$; in contrast, a positively charged surface diminishes osteoblast adhesion by approximately 20\% [7]. Meanwhile, regarding fibroblasts, Hamdan et al. report that both 
positively and negatively charged titanium surfaces promote fibroblasts adhesion, especially positively charged ones [60]. Their experimental results lead to the conclusion that a positively charged surface is detrimental for osseointegration of the titanium implant, because such a surface deters the bone-forming activity of osteoblasts, while at the same time promoting the fiber-forming activity of fibroblasts. In contrast, a negatively charged surface is beneficial for osseointegration overall, since it significantly promotes osteoblasts, while only slightly increasing fibroblasts adhesion. Fibroblasts adhesion to biomaterials is associated with variations in surface charge, and a parabolic relationship exists between surface charge and fibroblasts adhesion [61]. At relatively high and low surface charge densities, adhesion is relatively low. These evidences suggest that the influence of surface charge on fibroblast adhesion may be of primary importance for control of fibroblast/biomaterial interfacial behavior.

To explain the differences in the adhesion properties of osteoblasts and fibroblasts on the charged versus the neutral surface, the interaction between the surface of a biomaterial and the body fluid upon implantation in the human body must be understood. When an implant is inserted into the body, proteins adsorb on its surface within seconds, followed by cells, such as osteoblasts and fibroblasts, interacting with the proteins that have already been adsorbed, rather than with the actual implant material itself. The 
response of cells is strongly influenced by the adsorbed proteins and their conformations. The surface charge is considered to be one of the main physical factors that influence the integration process of the implant in the body [62]. One suggested explanation is that the charged implant surface could be different in the amount and / or the conformation of the adsorbed proteins from the neutral surface, which imparts different adhesion abilities to osteoblasts and fibroblasts [8]. However, the surface charge of a biomaterial does not remain the same in all the environments, but depends on the $\mathrm{pH}$ value and the composition of the surrounding fluids and the chemical composition of the biomaterial surface. Therefore, it is essential to understand the influence of surface charge and surface chemistry on the adsorption behavior of proteins, in order to improve the biocompatibility and long-term function of implants [62].

The point of zero charge (PZC) of the $\mathrm{TiO}_{2}$ layer that resides on the titanium dental implant surface is 6.8, which means that when the environmental $\mathrm{pH}$ equals 6.8 , the titanium implant has a neutral (i.e., zero) surface charge. When the value increases to physiological $\mathrm{pH}$ (typically 7.4), titanium dental implant with a $\mathrm{TiO}_{2}$ layer has a slightly negative surface charge [8]. On the other hand, in the physiological environment, the surface of HA is negatively charged, which partially explains why HA osseointegrates better than titanium. Another biocompatible material, $\mathrm{Al}_{2} \mathrm{O}_{3}$, has a PZC at 7.8, indicating 
that at physiological $\mathrm{pH}$, its surface is positively charged. In fact, when $\mathrm{Al}_{2} \mathrm{O}_{3}$ is used as implantation material, it always elicits a thick fibrous encapsulation, and fails to osseointegrate [8]. Table 1 summarises the relationship between bone bonding and surface charge for $\mathrm{HA}, \mathrm{TiO}_{2}$ and $\mathrm{Al}_{2} \mathrm{O}_{3}$.

Based on this theory, producing titanium dental implants with negatively charged surfaces may potentially improve the success rate for dental implantation. Currently, the theory of promoting titanium dental implant osseointegration through varying the implant surface charge is still at hypothetical stage. How to charge and hold these electric charges on the implant surface in clinical environment remains an open-ended question.

\section{Conclusion}

When implanted into human body, titanium dental implant needs to be osseointegrated with the host bone in order to achieve enough resistance against torsion caused by mastication. Surface conditions, such as surface roughness, surface charge, surface energy and composition have important influences on the osseointegration process. Therefore, modifying titanium implant surface seems to be a promising way to achieve stronger and faster osseointegration of the implants. Currently, surface roughening (e.g., SLA) and coating (e.g., with HA) are commonly used techniques in clinical practice. 
Both methods have their advantages and drawbacks as we have discussed in this paper. In addition, the surface charge of the titanium dental implant has been found to be directly related to its osseointegration. In conclusion, negatively charged surfaces are beneficial to the implant's osseointegration. Accordingly, the development of new methods that negatively charge the surface of a titanium dental implant seems to be a promising direction to improve osseointegration.

Although considerable research work has been done on developing and understanding titanium surface treatment methods, a number of important directions remain to be explored. For surface roughening, as we review in Section 3, nano-scale topography of the titanium dental implants is known to play a significant role in the osseointegration process. Yet, currently there are a few techniques for modifying the implant surface at such a fine level; moreover, most of these methods have only been tested in vitro. Concerning surface coating, much work remains to be done to incorporate bioactive drugs into the coating layer, in order to stimulate the growth of new bones onto the titanium dental implant, as well as to suppress the proliferation of bacteria. Finally, for surface charge modification, retention of the negative charge on the implant's surface remains to be an open-end problem. 


\section{Acknowledgements}

The authors would like to thank the Graduate School of the University of Hong Kong and the Prince Philip Dental Hospital for their continuous support. We also want to thank the John Wiley \& Sons and the Elsevier Inc. for their permission to reuse the images.

\section{References}

1. L. Le Guéhennec, A. Soueidan, P. Layrolle and Y. Amouriq, Dental Materials 23, 844-854 (2007).

2. J. Matthew, Titanium: A Technical Guide, Second Edition. ASM International (1988).

3. T. Eliades, Int. J. Oral Maxillofac. Implants 12, 621-627 (1997).

4. J. C. Keller, C. M. Stanford, J. P. Wightman, R. A. Draughn and R. Zaharias, J. Biomed. Mater. Res. 28, 939-946 (1994).

5. P. I. Brånemark, G. A. Zarb, and T. Albreksson, Plastic and Reconstructive Surgery 77, 496-497 (1986).

6. S. G. Steinemann, Periodontology 2000 17, 7-21 (1998).

7. J. Emsley, Nature's Building Blocks: An A-Z Guide to the Elements, pages 451-453. Oxford University Press (2001). 
8. M. Marcolonongo, N. J. Dinardo, N. Hickok, R. Tuan, K. Pourezzaei, R. Beard, D. Brennan, P. Heipp and T. Phan, in MRS Symp. Proc. 550, 121-126 (1999).

9. D. Buser, R. K. Schenk, S. Steinemann, J. P. Fiorellini, C. H. Fox and H. Stich, J. Biomed. Mater. Res. 25, 889-902 (1991).

10. L. Carlsson, T. Rostlund, B. Albrektsson, T. Albrektsson, Int. J. Oral Maxillofac. Implants 3, 21-24 (1988).

11. P. R. Klokkevold, P. Johnson, S. Dagrostari, A. Caputo, J. E. Davies and R. D. Nishimura, Clinical Oral Implants Res. 12, 350-357 (2001).

12. S. K. Nishimoto, M. Nishimoto, S. W. Park, K. M. Lee, H. S. Kim, J. T. Koh, J. L. Ong, Y. Liu and Y. Yang, Int. J. Oral Maxillofac. Implants 23, 675-680 (2008).

13. M. Schuler, G. R. Owen, D. W. Hamilton, M. deWild, M. Textor, D. M. Brunette and S. G. P. Tosatti, Biomaterials 27, 4000-4015 (2006).

14. R. Z. Legeros, Adv. Dental Res. 2, 164-180 (1988).

15. K. Bowers, J. Keller, B. Randolph, D. Wick and C. Michaels, Int. J. Oral Maxillofac. Implants 7, 302-310 (1992).

16. P. M. Brett, J. Harle, V. Salih, R. Mihoc, I. Olsen, F. H. Jones and M. Tonetti, Bone 35, 124-133 (2004). 
17. B. Chehroudi, T. R. L. Gould and D. M. Brunette, J. Biomed. Mater. Res. 23, 10671085 (1989).

18. Z. Schwartz, J. Y. Martin, D. D. Dean, J. Simpson, D. L. Cochran and B. D. Boyan, J. Biomed. Mater. Res. 30, 145-155 (1996).

19. A. Wennerberg, C. Hallgren, C. Johansson and S. A. Danelli, Clinical Oral Implants Res. 9, 11-19 (1998).

20. A. Wennerberg, T. Albrektsson, B. Albrektsson and J. J. Krol, Clinical Oral Implants Res. 6, 24-30 (1996).

21. W. Becker, B. E. Becker, A. Ricci, O. Bahat, E. Rosenberg, L. F. Rose, M. Handelsman and H. A. Israelsom, Clinical Implant Dentistry Relat. Res. 2, 159-165 (2000).

22. E. Lugscheider and T. Weber, IEEE Trans. Plasma Sci. 18, 968-973 (1990).

23. J. L. Ong, D. L. Carnes and K. Bessho, Biomaterials 25, 4601-4606 (2004).

24. M. Roccuzzo, M. Bunino, F. Prroglio and S. D. Bianchi, Clinical Oral Implants Res. 12, 572-578 (2001).

25. J. Deng, X. Zhang, P. Niu, L. Liu, J. Wang, Tribology International 39, 274-280 (2006). 
26. A. Wennerberg, T. Albrektsson and J. Lausmaa, J. Biomed. Mater. Res. 30, 251-260 (1996).

27. A. Gupta, M. Dhanraj and G. Sivagami, Intl. J. Dental Sci. 7, No. 1 (2009). http://www.ispub.com/journal/the_internet_journal_of_dental_science/volume_7_n umber_1_28/article/implant-surface-modification-review-of-literature.html

28. A. Wennerberg, T. Albrektsson, C. Johansson and B. Andersson, Biomaterials 17, 15-22 (1996).

29. C. Aparicio, F. J. Gil and A. Planell, J. Mater. Sci.-Mater. Med. 13, 1105-1111 (2002).

30. B. W. Darvell, N. Samman, W. K. Luk, K. F. Clark and H. Tideman, J. Dentistry 23, 319-322 (1995).

31. H. E. Placko, S. Mishra, J. J. Weimer and L. C. Lucas, Int. J. Oral Maxillofac. Implants 15, 355-363 (2000).

32. G. Orsini, B. Assenza, A. Scarano, M. Piatelli and A. Piatelli, Int. J. Oral Maxillofac. Implants 15, 779-784 (2000).

33. C. Aparicio, F. J. Gil, C. Fonseca, M. Barbosa and J. A. Planell, Biomaterials 24, 263-273 (2003). 
34. K. Gotfredsen, L. Nimb, E. Hjorting-Hansen, J. S. Jensen and Holmen, Clinical Oral Implants Res. 5, 202-206 (1994).

35. R. M. Palmer, R. J. Palmer and B. J. Smith, Clinical Oral Implants Res. 11, 179-182 (2000).

36. W. D. Müeller, U. Gross, T. Fritz, C. Voigt, P. Fisher, G. Berger, S. Rogaschewski and K. P. Lange, Clinical Oral Implants Res. 14, 349-356 (2003).

37. M. Piattelli, A. Scarano, M. Paolantonio, G. Lazzi, G. Petrone and A. Piattelli, J. Oral Implantology 28, 2-8 (2002).

38. S. Szmukler-Moncler, D. Perrin, V. Ahossi, G. Magnin and J. P. Bernard, J. Biomed. Mater. Res. 68, 149-159 (2004).

39. K. Yokoyama, T. Ichikawa, H. Murakami, Y. Miyamoto and K. Asaoka, Biomaterials 23, 2459-2465 (2002).

40. A. Wennerberg and T. Albrektsson, Int. J. Oral Maxillofac. Implants 15, 331-344 (2000).

41. D. Li, S. J. Ferguson, T. Beutler, D. L. Cochran, C. Sittig, H. P. Hirt and D. Buser, J. Biomed. Mater. Res. 60, 325-332 (2002).

42. D. L. Cochran, R. K. Schenk, A. Lussi, J. Biomed. Mater. Res. 40, 1-11 (1998). 
43. L. L. Hench and J. Wilson, An Introduction to Bioceramics. World Scientific, Singapore (1993).

44. K. de Groot, R. G. T. Geesink, C. P. A. T. Klein and P. J. Serekian, J. Biomed. Mater. Res. 21, 1375-1378 (1987).

45. Z. S. Leo, F. Z. Cui and W. Z., Li, J. Biomed. Mater. Res. 46, 80-86 (1999).

46. P. Ducheyne, W. van Raemdonck, J. C. Heughaert and M. Heughaert, Biomaterials 7, 97-103 (1986).

47. Z. S. Luo, F. Z. Cui and W. Z. Li, J. Crystal Growth 197, 939-943 (1999).

48. F. Li, Q. L. Feng, F. Z. Cui, H. D. Li and H. Schubert, Surface Coating Technol. 154, 88-93 (2002).

49. M. S. Block, J. N. Kent and J. D. Kay, J. Oral Maxil. Surg. 45, 601-607 (1987).

50. S. D. Cook, J. F. Kay, K. A. Thomas and M. Jarcho, Int. J. Oral Maxillofac. Implants 2, 15-22 (1987).

51. A. Jr. Sergio, E. Rumpel, E. Kauschke, J. Fanghänel and B. Jr. König, Annals of Anatomy 188, 143-151 (2006).

52. K. Søballe, Acta Orthopaedica 64, 1-58 (1993).

53. M. Weinlaender, E. B. Kenney, V. Lekovic, J. Beumer, P. K. Moy and S. Lewis, Int. J. Oral Maxillofac. Implants 7, 491-496 (1992). 
54. K. Søballe, E. S. Hansen, H. Brockstedt-Rasmussen and C. Bunger, J. Bone Joint Surgery 75B, 270-278 (1993).

55. T. W. Bauer, S. K. Taylor, M. Jiang and S. V. Medendorp, Clinical Orthopaedics and Relat. Res. 298, 11-18 (1994).

56. R. D. Bloebaum, D. Beeks, L. D. Dorr, C. G. Savory, J. A. DuPont and A. A. Hofmann, Clinical Orthopaedics and Relat. Res. 298, 19-26 (1994).

57. J. D. Jones, J. Lupori, J. E. Van Sickels and W. Gardner, Oral Surgery, Oral Medicine, Oral Pathology, Oral Radiology and Endodontology 87, 649-652 (1999).

58. K. A. Thomas, S. D. Cook, R. J. Haddad and K. L. Thomas, Proc. Annual Orthopedic Research Society Conference, p. 432 (1987).

59. N. Hamamoto, Y. Hamamoto, T. Nakajima and O. Hidehiro, Arch. Oral Biol. 40, 97106 (1995).

60. M. Hamdan, L. Blanco, A. Khraisat and I. F. Tresguerres, Clinical Oral Implants Res. 8, 32-38 (2006).

61. N. Hallab, K. Bundy, K. O’Connor, R. Clark, and R. Moses, Proc. Biomedical Engineering Conference, pp. 81-84 (1995).

62. I. Van De Keere, J. Vereecken and A. Hubin, Proc. Electrochemical Society Meeting, Abstract \#1873 (2009). 


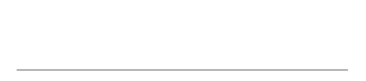

(a)

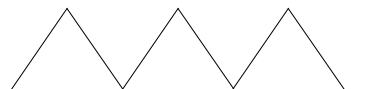

(b)

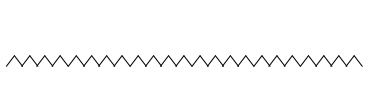

(c)

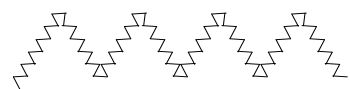

(d)

Fig. 1 Illustration of different levels of surface roughness: (a) Smooth surface; (b) Macrotopographically rough but microtopographically smooth surface; (c) Macrotopographically smooth but microtopographically rough surface; (d) Both macrotopographically and microtopographically rough surface. 


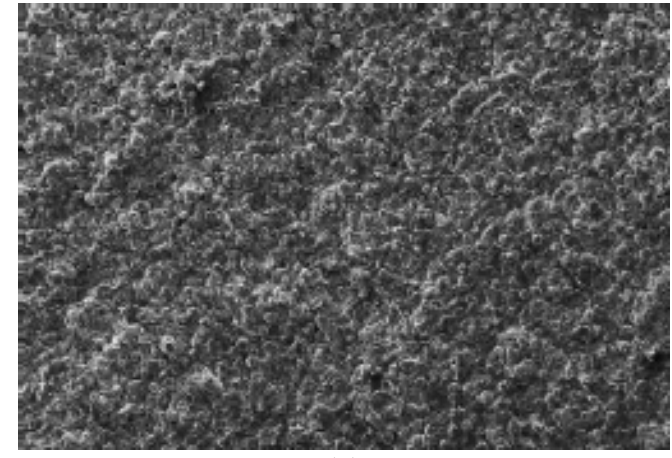

(a)

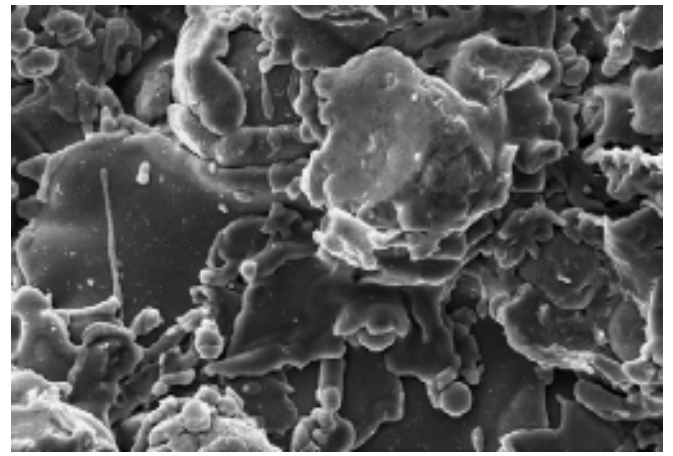

(b)

Fig. 2 SEM micrographs of a TPS surface. (a) ×50 magnification; (b) $\times 1000$ magnification [1] 


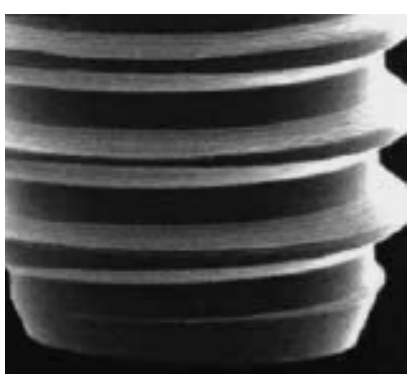

(a1)

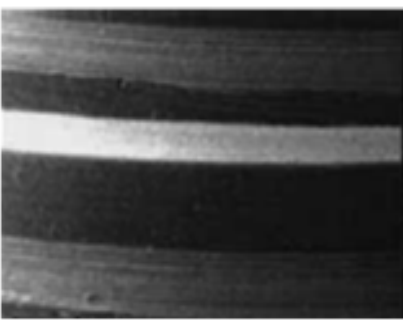

(a2)

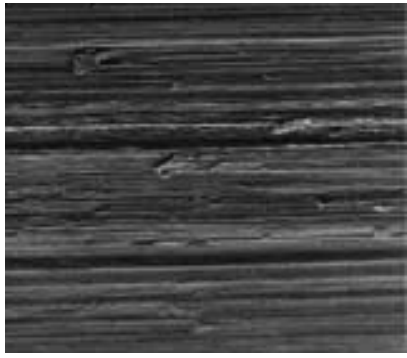

(a3)

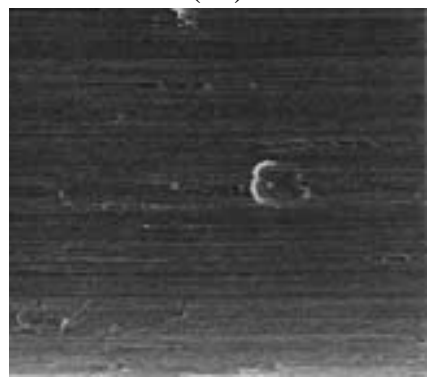

(a4)

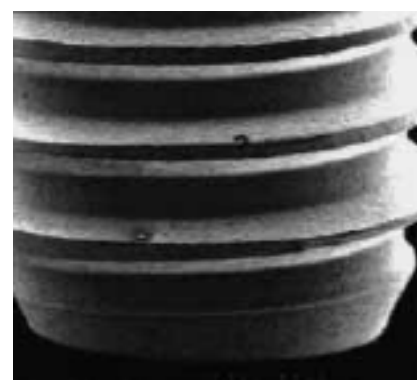

(b1)

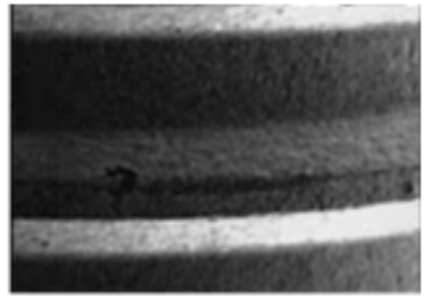

(b2)

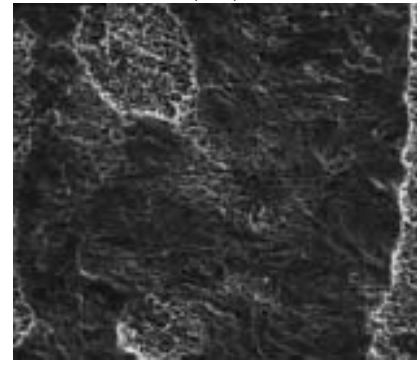

(b3)

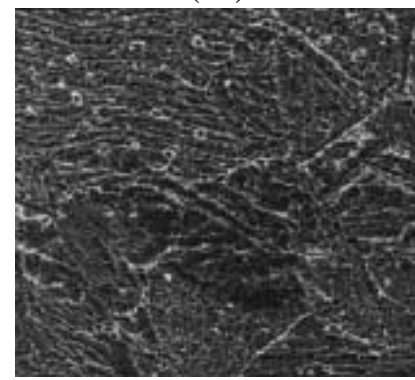

(b4)

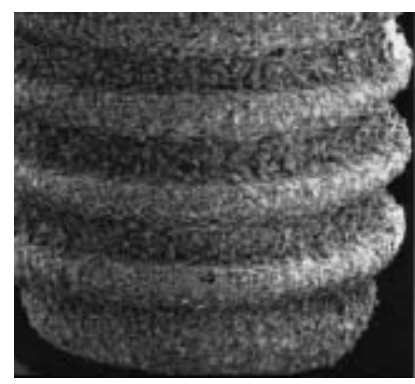

(c1)

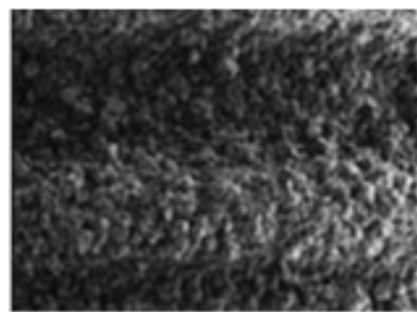

(c2)

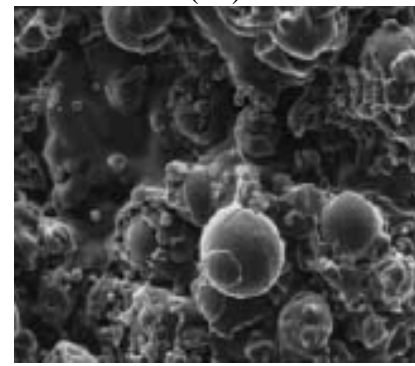

(c3)

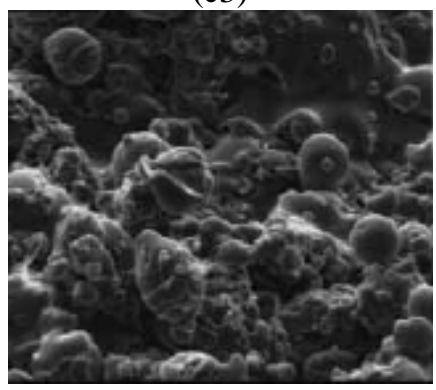

(c4)

Fig. 2 SEM images of different titanium dental implant surfaces. There are three types surfaces: unmodified, dual acid etched, and TPS-treated. Images in the same column are about the same type of surfaces, while those in the same row are produced using the same magnification. The first column (from left) images (i.e., a1-a4) show the unmodified implant surface, the second column (b1-b4) illustrate the dual acid etched surface, and the third (c1-c4) display the TPS surface. Among the rows, images in the first row (a1, b1, c1) are obtained using $\times 40$ magnification. The second row (a2, b2, c2) zoom to a higher $(\times 100)$ magnification, where the streaks are the peaks and valleys of the screw-shaped implant. The third row images $(a 3, b 3, c 3)$ zoom one of the thread peaks to $\times 1000$ mangification. The last row (a4, b4, c4) focus on one of the thread valleys, with the same magnification $(\times 1000)$ as the third row. Source: [11]. 


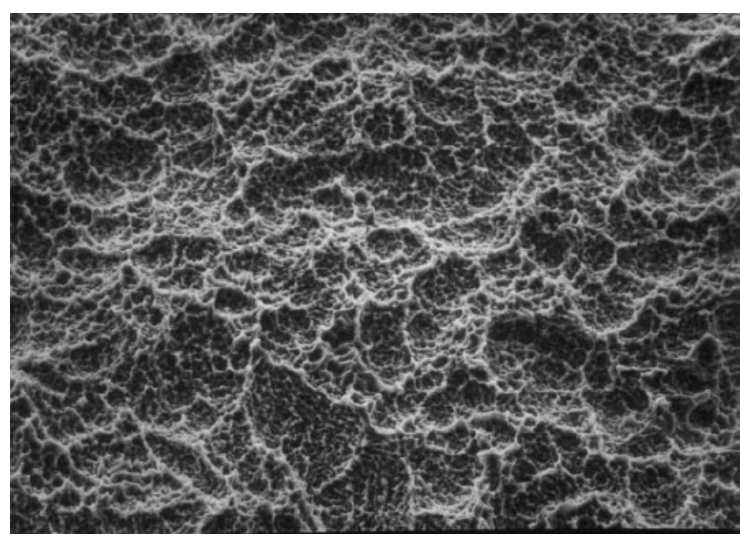

(a)

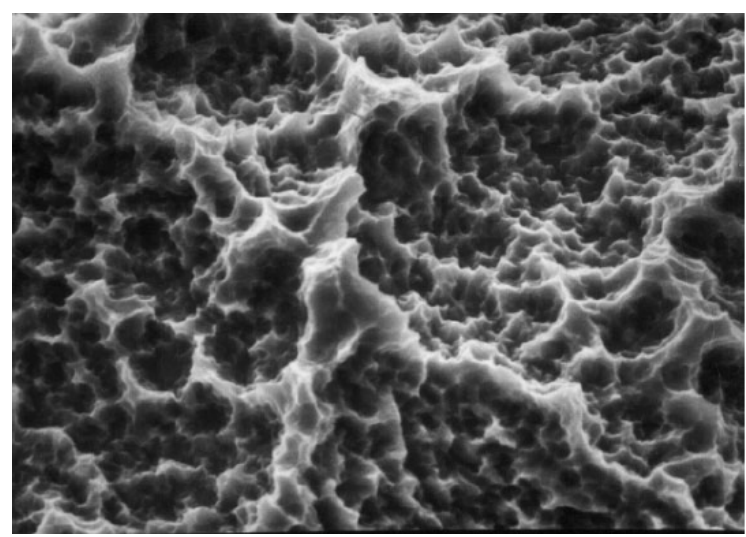

(b)

Fig. 3 SEM micrographs of an SLA implant. (a) Topography at $\times 500$ magnification. (b) Details of the topography at $\times 2400$ magnification. Source: [38]. 


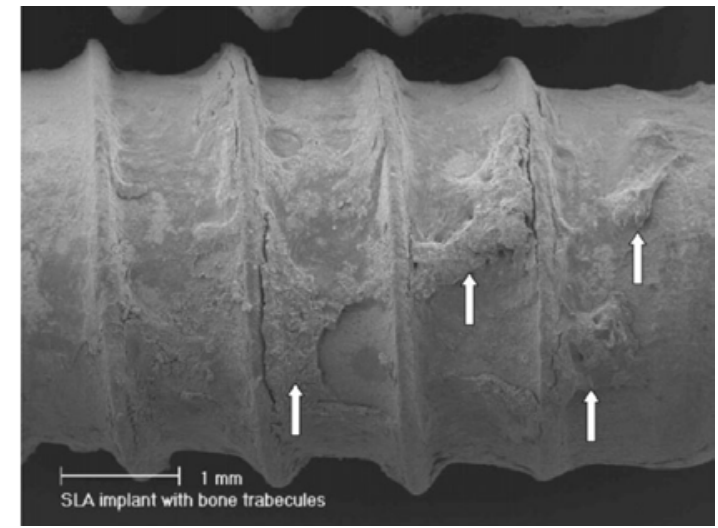

(a)

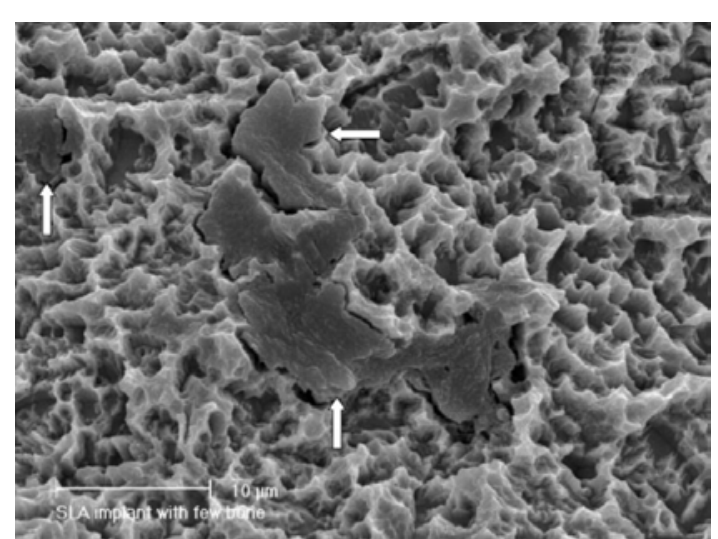

(b)

Fig. 4 Surface analysis of an SLA torqued implant. (a) Overview at $\times 15$ magnification. (b) Detail of the attached bone at $\times 6000$ magnification. Source: [38]. 


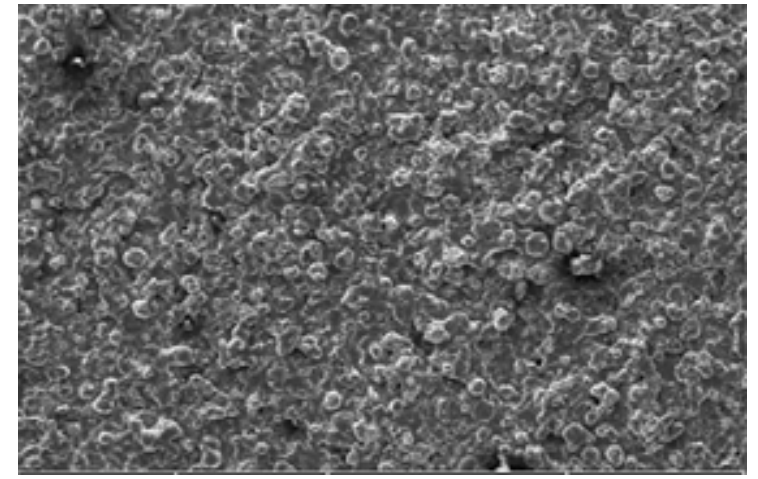

(a)

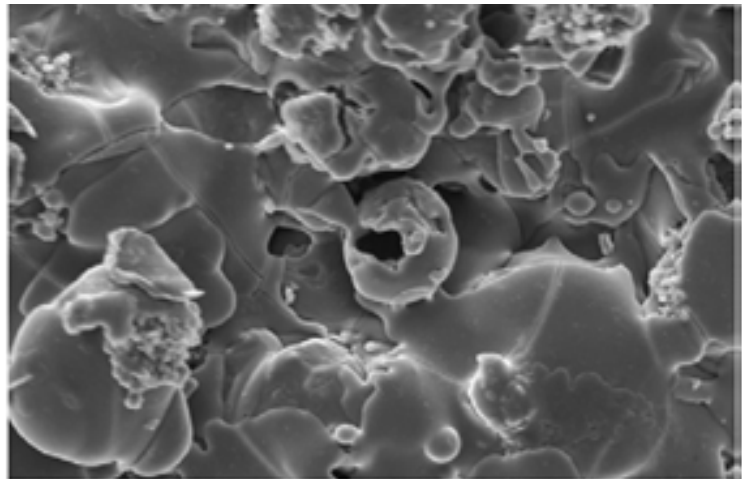

(b)

Fig. 6 SEM micrographs of a plasma-sprayed HA coating surface: (a) $\times 100$ magnification, (b) $\times 1000$ magnification. Source: [1]. 
Table 1 Relationship between bone bonding and surface charge for three biomaterials [7]

\begin{tabular}{|c|c|c|}
\hline Biomaterial & Bone Bonding & Surface charge \\
\hline $\mathrm{HA}$ & Strong & Negative \\
\hline $\mathrm{TiO}_{2}$ & Slightly Strong & Slightly Negative \\
\hline $\mathrm{Al}_{2} \mathrm{O}_{3}$ & Weak & Positive \\
\hline
\end{tabular}

